\title{
A User's Guide to the Hydrogen Monitor
}

\author{
M. M. Meier* \\ E. L. Adams
}

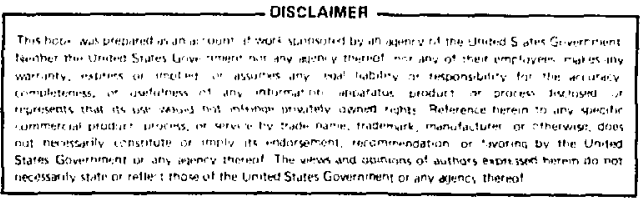

* National Bureau of Standards, Washington, DC 20234.

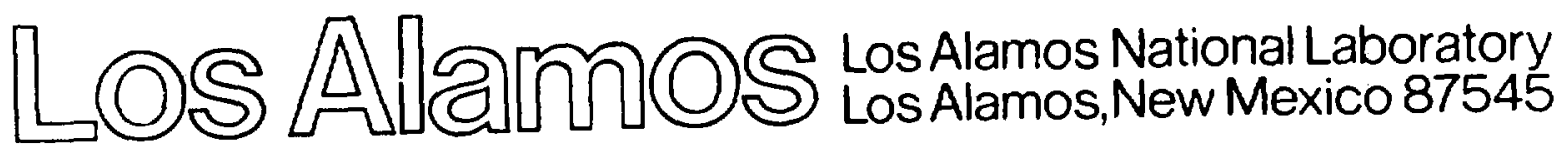




\title{
A USER'S GUIDE TO THE HYDROGEN MONITOR
}

\author{
by
}

M. M. Meier and E. L. Adams

\section{AESTRACT}

A protocol for calibration of the hydrogen monitor and for its use as an assay instrument is presented. A statistical analysis for the system, including effects of deadtime and pile-up loss, is developed, and the software that supports the system is described.

\section{INTRODUCTION}

The software developed and the procedures to be used for calibration and running of the ${ }^{252} \mathrm{Cf}$ hydrogen monitor (HM) are documented in this guide. Computer software was developed for use with the Digital Equipment Corporation (DEC) LSI-11 using the Version 3 operating system. Potential users are also referred to Refs. 1 and 2, which describe the ${ }^{252} \mathrm{Cf}$-based HM.

\section{EXPERIMENTAL}

Design details of the $H M$ are given in Ref. 1. Figure 1 shows the electronics block diagram used in the assay of High-Temperature Gas-cooled Reactor (HTGR) "green" rods, December 1979. The data were accumulated in a multichannel analyzen (MCA) for constant live time. The spectrum of Fig. 2 is 


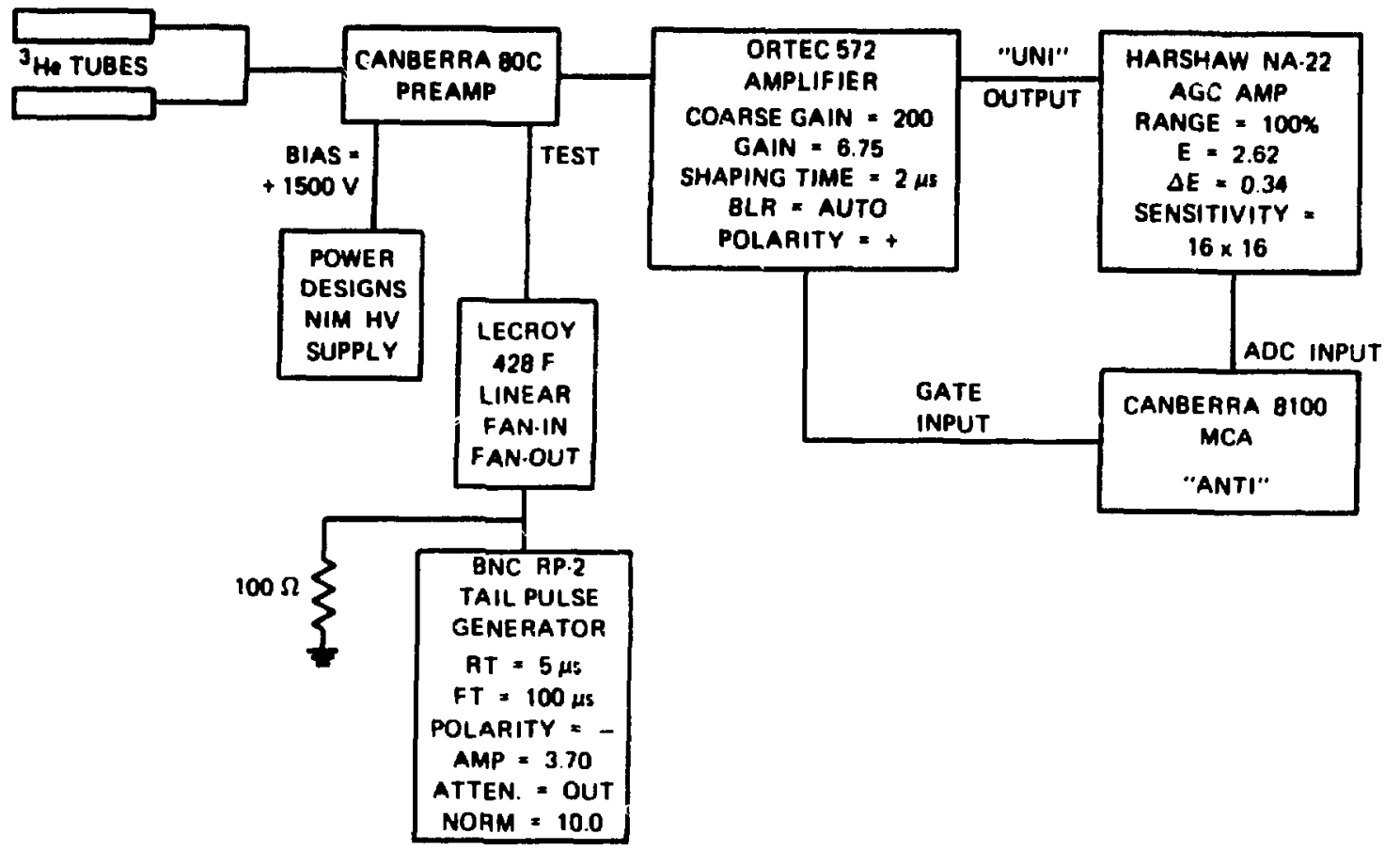

Fig. 1 .

Hydrogen monitor electronics.

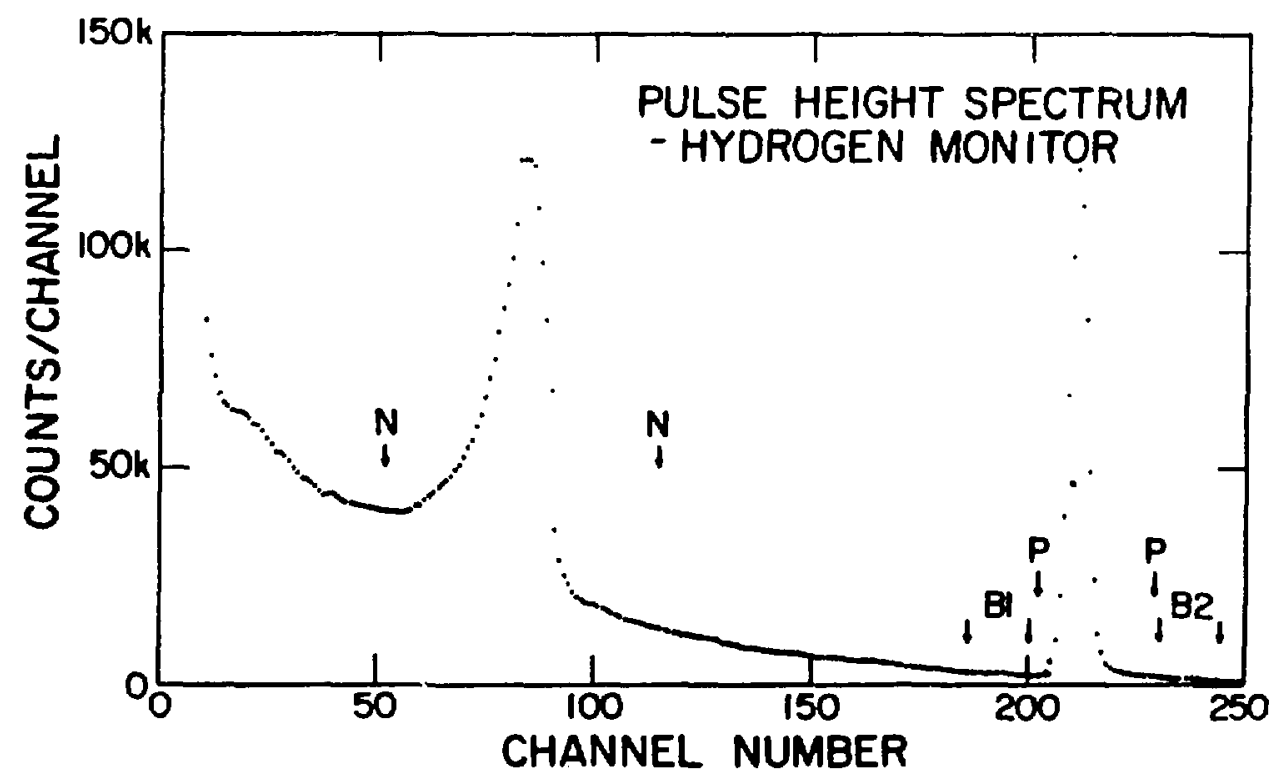

Fig. 2 .

Typical pulse-height spectrum from the hydrogen monitor. The data of interest are the integrated regions $N, B 1, P$, and $B 2$, as discussed in the text. 
representative for a live time of $800 \mathrm{~s}$. Summations for the spectrum intervals indicated were printed out for each run. They rep.esent, respectively, events in the thermal peak, $N$; low pulse height backg.ound for the pulser peak, B1; pulser peak plus background, $P$; and high puise height background for the pulser peak, B2. Intervals for pulser peak and background are selected so that net pulser peak area, $P_{0}$, equals $P-(B 1+B 2)$. The four indicated sums were printed out at the end of each run and comprised the raw data.

\section{REDUCTION OF DATA TO RELATIYUE RESH ONSES}

Relative responses, defined as the ratio of the true counts in the thermal peak for an unknown or standard sample to counts for an enipty chamber ("background"), were the necessary data for calibmation and use of the HM.

The thermal peak is used as a referen amplitude for the gain stabilization circuitry indicated in Fig. 1. Thus, when bias voltage drift.s occur, the thermal peak amplitude remains constant, but the pulser peak may exhibit some drift. For this reason, as well as the inherent decay of the ${ }^{252} \mathrm{Cf}$, the background for each sample is a function of time. Therefore, for a series of runs that include several background runs, and for which pulser peak drift is not so large as to necessitate changing the $B 1, B 2$, and $P$ windows, the backgrounds are $f$ it to a linear function of $t$ ime.

The procedure followed was to treat the first background run as a reference for normalization. The reduced pulser peak, $P_{0}$, for each background run was calculated and the ratio of each to the first was determined. Then the true rate for the ith background run was proportional to

$$
N_{0}(i)=N(i) /\left[P_{0}(i) / P_{0}(1)\right] .
$$

The relative uncertainty is

$$
\left[\frac{\Delta N_{0}(i)}{N_{0}(i)}\right]^{2}=\left[\frac{\Delta N(i)}{N(i)}\right]^{2}+\left[\frac{\Delta P_{0}(i)}{P_{0}(i)}\right]^{2}+\left[\frac{\Delta P_{0}(1)}{P_{0}(1)}\right]^{2}
$$


and

$$
\left[\Delta P_{0}(i)\right]^{2}=[\Delta P(i)]^{2}+[\Delta B 1(i)]^{2}+[\Delta B 2(i)]^{2}
$$

with

$$
\Delta B 1(i)=\sqrt{B 1(i)},
$$

and

$$
\Delta B 2(i)=\sqrt{B 2}(i),
$$

as usual, but the statistical uncertainty in $P(i)$ is only dependent on the number of lost counts that would have appeared in the peak. For example, consider the loss rate determination as an experiment in which a random source is counted only when the periodic pulse is present. The number of events counted in such an experiment is exactly the lost number in ours, and it is this quantity on which the statistical uncertainty depenos. Therefore, even though only relative loss rates enter the calculation of relative response, knowledge of absolute loss rate is needed for calculation of the uncertainty.*

Each run was assigned a sequential identification number that was approximately proportional to time after the first run. This identificaion number was used as the time parameter for the linear least squares $f$ it of the response to a straight line.

The program LSQMKl was used for the fitting procedure. This program requires input responses, uncertairities, and time paraneters on the of $C$ default file device (DK:), which can be conveniently created using the DEC-

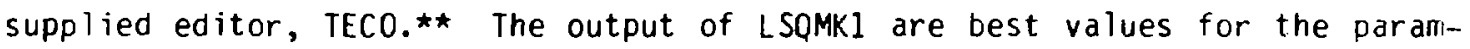
eters $A 1$ and $A 2$ of the background parameterization

$$
B K(T)=A 1+A 2 \cdot T,
$$

* We determined the absolute loss rate by measuring the pulser rate on an oscillascope as $(1040 \pm 40 \mu \mathrm{s})^{-1}=960 \mathrm{cts} / \mathrm{s}$. The total number of counts in 800 "live" seconds ( 890 true seconds) was then 850000 . Observed pulser counts were 625000 and total loss was 225000 counts. Therefore $\Delta P=470$; $\angle P_{0} / P_{0}=0.08$ per cent.

$\rightarrow$ is an aid in preparing the input file and interpreting the results, the - rsductory commentary for LSQMKl and a typical output page are reproduceo : Errictated in Appendix A. 
the estimate of the three-parameter variance-covariance matrix that describes the uncertainty in the background,

$$
\left[\begin{array}{ll}
A_{3} & \\
A_{4} & A_{5}
\end{array}\right],
$$

where

$$
[\Delta B K(T)]^{2}=A 3+2 A 4 T+A 5 T^{2},
$$

and $x^{2}$ /degree of ireeoom $\left(x^{2} / \mathrm{deg}\right)$. For $2^{2} / \mathrm{deg} \geq 1.0$, the $\mathrm{fit}$ is considered reasonable. When $x^{2} /$ deg exceeds 1.0 significantly, it is reasonable to multiply the variance-covariance matrix by $x^{2}$ /deg to obtain a more realistic estimate of $\triangle B K(T)$. A preferable course of action, of course, is to find and eliminate the systematic error that is responsitle for the effect. If the assumption of linearity of the function is not valid, though, the former course of action may be the only one practical.

The relative response, $R(T)$, for a sample with run identification $T$ $(\mathrm{t}$ ime $=\mathrm{T})$ is then

$$
\begin{aligned}
R(T)= & {[N(T) / B K(T)]\left[P_{0}(1) / P_{0}(T)\right], } \\
{[\Delta R(T) ! R(T)]^{2}=} & 1 / N(T)+[\Delta B K(T) / E K(T)]^{2}+\left[\Delta P_{0}(T) / P_{0}(T)\right]^{2} \\
& +\left[\Delta P_{0}(1) / P_{0}(1)\right]^{2}
\end{aligned}
$$

As before,

$$
\left[\Delta P_{0}(T)\right]^{2}=E 1(T)+E 2(T)+N_{\text {Lost }}
$$

where "lost is the number of lost pulser pulses.

A program, "H Monitor Response," has been written and recorded on magnetic card for calculation of response and uncertainty from the linear background 
function $B K(T)$ using the HP-67 calculator. This program assumes that an LSQMK1 $f$ it for $B K(T)$ has already been done and that the parameter estimates $A_{1}$ - $A_{5}$ are available. This program is described in Appendix $B$.

When drifts occurred such that the pulser peak and background windows needed to be changed, the run sequence was restarted with a new background and reference run. Confidence in the technique was bolstered by reproducing responses within more than one sequence.

\section{RELATIVE MEASUREMENT FORMALISM}

In the region of interest, the response of the hydrogen monitor can be represented by the expression

$$
R=\sum_{i=1}^{n} A_{i} x_{i},
$$

where $x_{1}=1.0$ and the remaining $x_{i}$ 's are the contents of a sample, iricluding hydrogen, which perturb the response. The calibration problerr iies in deciding which sample characteristics $\left(x_{j}\right)$ produce the largest perturbations, fabricating standards with these characteristics accurately known, ano determining the $A_{i} s$ by accurate measurement of the standards responses anc least squares fitting of them. In principle, the instrument can then be used to measure hydrogen in unknowns through the relation

$$
H^{U}=\left(R^{U}-\sum_{\substack{i=1 \\ i \neq H}}^{N} A_{i} x_{i}^{U}\right) / A_{H},
$$

as long as the perturbing constituents $x_{i}{ }^{U}$ are closely enough matched to the constituents of the standards that their respective effects on the response are approximated by the assumed linear relationship. The uncertainty in the result of such an assay is 


$$
\begin{aligned}
o\left(H^{U}\right)^{2}= & A_{H}{ }^{-2}\left[\partial\left(R^{U}\right)^{2}+\sum_{i \neq H} A_{i}^{2} o\left(x_{i}\right)^{2}\right. \\
& +\sum_{i \neq H} \sum_{j \neq H} x_{i}^{U} x_{j}^{U} \operatorname{cov}\left(A_{i}, A_{j}\right) \\
& +2 H^{U} \sum_{i \neq H} x_{i}^{U} \operatorname{cov}\left(A_{i}, A_{H}\right) \\
& \left.+\left(H^{U}\right)^{2}\left(A_{H}\right)^{2}\right],
\end{aligned}
$$

where $\sigma(Y)$ is the standard deviation (I sigma) associated with the variable $Y$, and $\operatorname{cov}\left(A_{i}, A_{j}\right)$ is the element of the variance-covariance matrix carespending to the covariance of parameters $A_{i}$ and $A_{j}\left(\operatorname{cov}\left(A_{j}, A_{j}\right)\right.$ $\left.o\left(A_{j}\right)^{2}\right)$. The uncertainty is written in the above form to show its explicit dependence on $\sigma\left(A_{H}\right)^{2}$ and $\operatorname{cov}\left(A_{j}, A_{H}\right)$.

A second technique for determining hydrogen with this instrument is to run standards along with unknown samples. In this case

$$
H^{U}=H^{S}\left(R^{U}-\sum_{i \neq H} A_{i} x_{i}^{U}\right) /\left(R^{S}-\sum_{i \neq H} A_{i} x_{i}^{S}\right),
$$

where $H^{S}$ is che hydrogen content of the standard, $R^{S}$ is its response, and $x_{i}^{S}$ its perturbing constituents. For this case, the uncertainty is

$$
\begin{aligned}
\sigma\left(H^{U}\right)^{2}= & {\left[\left(H^{S}\right)^{2} / D(S)^{2}\right]\left[\sigma\left(R^{U}\right)^{2}+\sum_{i \neq H} A_{i}{ }^{2} \sigma\left(x_{i}\right)^{2}+\left(H_{U} / H_{S}\right)^{2} \sigma\left(R^{S}\right)^{2}\right.} \\
& +\left(H_{U} / H_{S}\right)^{2} \sum_{i \neq H} A_{i}{ }^{2} \sigma\left(x_{i} S^{2}\right]+[D(U) / D(S)]_{\sigma}{ }^{2}\left(H^{S}\right)^{2} \\
& +\sum_{i \neq H} \sum_{j \neq H}\left[x_{i} U / D(U)-x_{i} S / D(S)\right]\left[x_{j}^{U} / D(U)-x_{j} S / D(S)\right] \operatorname{cov}\left(A_{i}, A_{j}\right) .
\end{aligned}
$$

7 
with $D(T)=R^{\top}-\sum_{i \neq H} A_{i} x_{i}^{T}$. Note the absence of dependence on variances or covariances of $A_{H}$ and that for well-matched unknowns and samples $\left(x_{i}^{U} \simeq x_{i}^{S}\right)$ the coefficient of $\operatorname{cov}\left(A_{j}, A_{j}\right)$ tends to vanish.

V. CALIBRATION

A. General

From preceding and preliminary work it is necessary to determine which sample characteristics can be expected to perturb the response of the $H M$. These considerations then lead to the design and fabrication $c$ standards in which the major characteristics are varied over the ranges to be expected the unknowns. The standards then are assayed with high precision in the HM, and the responses, uncertainties, and quantitative characteristics of the standards form the data base for least squares fitting to produce the calibration constants.

The calibration of the HM for the 1979 assay of HTGR rods serves as an example of this procedure. Earlier work, 1 indicated a dependence of the Hr: response on ${ }^{235} \mathrm{U}$ content and a nonlinear dependence on hydrogen content. In addition, preliminary work showed a strong sensitivity to the sample position. For these reasons, standards were fabricated with the variable hydrogen, ${ }^{235} U$, and fill height, each in the range of interest of the HTGR rocs. They are listed in Table I.

TABLE I

CALIBRATION STANDARDS FOR HYDROGEN MONITOR

\begin{tabular}{|c|c|c|c|c|c|c|c|}
\hline $\begin{array}{c}\text { Standarn } \\
\text { ID } \\
\end{array}$ & $\begin{array}{l}H \text { Contents } \\
\text { (g) }\end{array}$ & $\underline{\text { Cap }}$ & $\begin{array}{c}\text { Sapple } \\
\text { Height (cm) }\end{array}$ & $\begin{array}{c}{ }^{235} \mathrm{u} \text { Contents } \\
(\mathrm{g})\end{array}$ & Comments & Average Response & $x^{2}$ \\
\hline $\begin{array}{l}1 \\
2 \\
3 \\
4 \\
5 \\
6\end{array}$ & $\begin{array}{l}0.5768 \\
0.6420 \\
0.5713 \\
0.6467 \\
0.5696 \\
0.6306\end{array}$ & $\begin{array}{l}1.0 \\
1.0 \\
1.0 \\
1.0 \\
1.0 \\
1.0\end{array}$ & $\begin{array}{l}4.31 \\
4.31 \\
4.31 \\
4.31 \\
4.31 \\
4.31\end{array}$ & $\begin{array}{l}0.1036 \\
0.0920 \\
0.1873 \\
0.1916 \\
0.2930 \\
0.2782\end{array}$ & $\begin{array}{l}\text { Cra-l analytic } \\
\text { chemistry samples } \\
\text { encapsulated in } \\
\text { polyethylene vials. }\end{array}$ & $\begin{array}{l}1.5004 \neq 0.0012 \\
1.5839 \neq 0.0013 \\
1.4991 \neq 0.0012 \\
1.5855 \neq 0.0013 \\
1.4972 \neq 0.0012 \\
1.5719 \neq 0.0012\end{array}$ & $\begin{array}{l}28.10 \\
4.81 \\
1.36 \\
0.73 \\
1.25 \\
9.26\end{array}$ \\
\hline $\begin{array}{l}P-1 \\
P-2\end{array}$ & $\begin{array}{l}0.4396 \\
0.5004\end{array}$ & $\begin{array}{l}0.0 \\
0.0\end{array}$ & $\begin{array}{l}5.00 \\
5.00\end{array}$ & $\begin{array}{l}0.0 \\
0.0\end{array}$ & $\begin{array}{l}\text { Weighed poly- } \\
\text { ethylene vials. }\end{array}$ & $\begin{array}{l}1.3115 \neq 0.0011 \\
1.3712 \neq 0.0011\end{array}$ & $\begin{array}{l}0.06 \\
0.07\end{array}$ \\
\hline$p+$ Cap & 0.4983 & 1.0 & 5.00 & 0.0 & $\begin{array}{l}\text { Encapsulation for } \\
\text { CMB-1 stanaards }\end{array}$ & $1.4238 \div 0.0014$ & 16.19 \\
\hline
\end{tabular}


B. Standards

Standards 1 through 6 were fabricated by the Los Alamos Analytical Chemistry Group (CMB-1) and are comprised of $\mathrm{U}_{3} \mathrm{O}_{8}$ mixed with dextrose and graphite. The ${ }^{235} \mathrm{U}$ content was nominally 160,200 , and $300 \mathrm{mg}$ with quoted accuracies of \pm 0.01 per cent and the hydrogen content was 100 and $150 \mathrm{mg} \pm 0.01$ per cent. The mixture's primary encapsulation was a glass "1-dram" vial with a Bakelite cap. Sufficient graphite was added to each standard to give it a constant $(20 \pm 1 \mathrm{~mm})$ fill height. To make the resemblance to HTGR rods closer, a secondary encapsulation of a "2-dram" polyethylene vial, similar to those that provide primary encapsulation for the HTGR rods, was provided for each standard. The hydrogen content for each of these vials was oetermined by weighing and multiplying by the stoichiometric mass ratio, which was taken to be 0.1437 . The mass determination by weighing was done on a calibrated commercial scale and has an accuracy of $\pm 0.2 \mathrm{mg}(0.03 \mathrm{mg}$ hydrogen). The hydrogen content in the table is the sum of the polyethylene hyorogen and dextrose hydrogen. The uncertainty was taken to be $\pm 0.1 \mathrm{mg}$, an estimate of our uncertainty in the stoichiometry.

The Bakelite cap contains hydrogen that is not uniformily distributed in the sample and that does not appear in the HTGR rods. To account for this characteristic we assign to these standards a parameter that is equal to 1.0 when the cap is present and 0 when it is not. A random sample of ten caps was weighed and found to have a relative standard deviation in mass of 0.87 per cent. The uncertainty in this parameter was therefore assigned the value 1.0 per cent. The height of the "2-dram" vials was about $5.0 \mathrm{~cm}$ with nominal contents of $450 \mathrm{mg} \mathrm{H}$, and the fill height of the glass vials was $2.0 \mathrm{cmi}$ with nominal contents of $150 \mathrm{mg} \mathrm{H}$. The fill height listed in the table is the weighted average for the encapsulated sample and is assigned an uncertainty of $0.1 \mathrm{~cm}$.

Standards $P-1$ and $P-2$ are simply weighed polyethylene vials. Standard "P + Cap" is an empty glass vial with a cap and polyethylene encapsulation.

C. Experimental Procedure

Each standard of Table $I$ was assayed three times over one continuous period and their responses were calculated as described above. Weighted averages were calculated for the response of each standard using either AVERGE or LSQMK1. AVERGE is a program designed to take teletype input values and uncertainties and calculate their weighted average, standard deviation of the mean, relative 
standard deviation, and $x^{2} /$ deg. It was interesting to recora relative responses, uncertainties, and time parameter on floppy disk for LSQMK1 input. In this way, the above information was generated by doing a single (constant) parameter $f$ it to the data. One could then look for residual time dependence of the response (which was not removed by the BK(T) technique) indicating a systematic source of error by attempting a fit to a linear function of tinie. No residual time dependence was found in instances where this technique was used. The weighted averages for each response are listed in the table. A measure of the reproducibility of the system is $x^{2}$ /degree of freedom $\left({ }^{2} /\right.$ deg) for these averages. They ranged fron a low of 0.10 to a high of 1.34 with a simple average of 0.71 , giving confidence that the experiniental variations were statistical.

D. Fitting of Data

Linear least squares fits were oone to obtain best values for the parameters $A_{j}$, the associated variance-covariance matrix, and the value of $x^{2} /$ dey for each fit. LSQMKl was used for this operation, the responses, uncertainties, and perturbing constituents of the standards having been recorded un disk with TECO. Parameters that were considered to represent the characteristics of the samples were hydrogen content, fill height, Bakelite cap, and ${ }^{235} U$ contents. Although not strictly correct from a statistics point of view, a term for hydrogen-squared content was included as an independent parameter. Such a procedure is certainly not valid for situations where this tern becomes comparable to, or larger than, the linear term but may be a reasonable way to include such effects if they are small perturbations on the response.

The fitting procedure of LSQMK1 assumes negligible uncertainties in the specification of the perturbing constituents. This is not always the case, and the uncertainty in the response can be enlarged to take account of this to first order. After obtaining a fit with the unmodified response, the functional form of the response is

$$
R=\sum_{i=1}^{N} A_{i} x_{i}, x_{1}=1.0 .
$$

For uncertainties, $\Delta x_{j}$, in the $x_{i}$ 's that are not negligible, the response uncertainty for a given standard can be expressed 


$$
\begin{aligned}
\sigma\left(R^{S}\right)^{2} & =\sigma_{S t a t}\left(R^{S}\right)^{2}+\sum_{i=1}^{N}\left(\frac{\delta R}{\delta x_{i}^{S}}\right)^{2} a\left(x_{i}^{S}\right)^{2} \\
& =\sigma_{\text {Stat }}\left(R^{S}\right)^{2}+\sum_{i=2}^{N} A_{i}^{2} o\left(x_{i}^{S}\right)^{2},
\end{aligned}
$$

where a ${ }_{S t a t}\left(R^{S}\right)^{2}$ is the unmodified variance dependent only on the counting statistics of the response.

The nine standards' responses were the data to be $f i t$, and the parameter sets were varied to see the effect on $x^{2} /$ deg, reasonableness of parameters, and resulting assay accuracy. Table II gives a list of parameter sets obtained with $x^{2}$ /deg less than 30. Trial assays were done with each of the sets to find overall uncertainties that result from their use. In order to have the assay uncertainties reflect the imperfect fit of the linear model to the calibration data, the variance-covariance matrix associated.wth each set was multiplied by $x^{2} /$ deg. Two objections to using the set with the smallest $x^{2} /$ deg arise. First, the sign of the coefficient of fill height is physically unreasonable. One experimentally observes the response of the instrument to decrease as the hydrogen is moved from its central, optimum position. Hence a decrease in response is expected as the height of the sample is increased, which would give a negative sign to the coefficient of fill height. A second problem is the increase in the assay uncertainty, probably caused by the addition of a quantity that has a relatively large variance itself. To reduce the height uncertainty much below its $1-\mathrm{mm}$ level would involve careful consideration of the weighted averaging of polyethylene and dextrose heights. For these

TABLE II

\begin{tabular}{|c|c|c|c|c|c|c|c|}
\hline Set & $x^{2} / \operatorname{deg}$ & $A_{1}$ & $\mathrm{~A}_{2}\left(\mathrm{H}, \mathrm{g}^{-1}\right)$ & $A_{3}(C a p)$ & $A_{4}\left(H^{2}, g^{-2}\right)$ & $\mathrm{A}_{5}\left(\mathrm{Ht}, \mathrm{cm}^{-1}\right)$ & $\mathrm{H}(\mathrm{mg})$ \\
\hline H8М309 & 16.3 & 0.818 & 1.114 & 0.047 & -- & -- & 3.4 \\
\hline H8M4O9 & 12.4 & 0.833 & 1.046 & 0.045 & 0.078 & -- & 3.5 \\
\hline H8M509 & 6.2 & 0.719 & 1.078 & 0.049 & 0.103 & 0.019 & 4.2 \\
\hline
\end{tabular}

HYDROGEN MONITOR CALIBRATION PARAMETERS 
reasons, the set H8M409 was chosen as representing the best parameters and variance-covariance matrix.

\section{UNKNOWNS ASSAY AND ANALYSIS}

To obtain the hydrogen content of the HTGR rods, they were assayed in the hydrogen monitor along with two standards for each blend. The sequence was chamber empty, first standard, four sequential rods, second standard. The standards associated with the various blends were blend 12, standards 2 and 4; blend 13, standards 2 and 4 ; and blend 14, standards 4 and 6 . This procedure gave four to seven replicate assays of each standard for each day, permitting an assessment of system stability. Average responses, * their uncertainties, and $x^{2} /$ deg for each standard were calculated for each continuous running period. $x^{2} /$ deg greater than 2.0 occurred for 40 per cent of the cases, arid for these the uncertainty of the average response was increased by a factor of

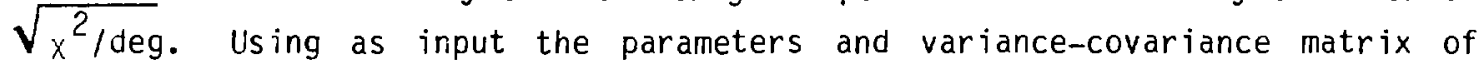
set $18 \mathrm{M409}$, the average responses of the standards, and the response*t of each unknown, the hydrogen content of each of the latter was computed relative to the two standards. The program used for the calculation was LINREL, which computes the hydrogen contents of the rod by Eq. (12). LINREL requires three floppy disk files for input. The first is the set of best parameters and associated variance-covariance matrix produced by the calibration procedure. These were dumped during the LSQMK1 runs for the satisfactory calibration sets. A slight modification of these dumps with TECO makes them compatible with the LINREL input. The second file includes average responses and characteristics of the standards that were run in conjunction with the unknown samples. This file must be generated to be consistent with the order of variables in the LSQMK1 output and includes, explicitly, the uncertainties in these perturbing constituents. Finally a file including unknowns' responses, uncertainties, and constituents' values (in the LSQMKI order) is input. The introductory comments for LINREL are reproduced in Appendix $C$ in order to elucidate the structure of the input files. A typical output is also included to

*Average values and $x^{2} /$ deg were computed using AVERGE.

**Responses were calculated using the technique discussed above, i.e., generation of a function BK(T) with LSOMK1 and calculation with the HP-67 program. 
aid in the interpretation of the results. The constituents of the standards and their uncertainties are discussed in Sec. III. The constituents of the rods were taicen to be Cap $=0.0 \pm 0.01, H^{2}=0.400 \pm 0.010 \mathrm{~g}^{2}$, and Height $=5.00 \pm 0.100 \mathrm{~cm}$. The values for hydrogen were the weighted averages of the two determinations relative to the standards. These differed from the values obtained relative to each standard by about $3 \mathrm{mg}$, which was also the statistical accuracy of the determination. Since the nominal $H^{2}$ value was valio only for total hydrogen contents of $0.6325 \mathrm{~g}$, an analytic expression was used to correct the hydrogen with an $\mathrm{H}^{2}$ correction bised on the initial assay results. Several cases were run using an iterative procedure to arrive at selfconsistent values of $H$ and $H^{2}$. These agreed with the analytically generated correction to better than $0.3 \mathrm{mg}$.

\section{CONCLUSIONS}

The hydrogen monitor has been calibrated for measurements in the vicinity of $600-\mathrm{mg}$ hydrogen. With the current calibration and standards, the estimated accuracy is $\pm 4.5 \mathrm{mg}$, which is based on the difference in assay results for the same unknown for two different standards and measurement precisions. Before proceeding with work that improves the precision of the monitor, more work will need to be done to remove this $\pm 3-m g$ discrepancy. Likely candidates for investigation are the exact form of the response, which is likely to be more complex than the simple linear response assumed here, and use of calibration standards, which more closely match the rods (perhaps by elimination of the Bakelite cap).

Another matter of concern is the nigh position sensitivity of the HM. It would be convenient to redesign the instrument for more uniform irradiation of the sample, especially over its vertical dimension. Currently a 1-mm shift in position alters the response by 5 per cent, inducing one to believe that fill height must be an important parameter.

There may be correlations in the calibration data and the standards" responses that we have not properly treated. To take account of the constituents' uncertainties in the calibration, it was necessary to expand the response uncertainty by the additional term 


$$
\sum_{i=1}^{N}\left(\frac{\delta R}{\delta x_{i}}\right)^{2} \Delta x_{i}{ }^{2}
$$

If the same standards are used in the relative measurements, then the uncertainties for these two measurements are not independent, although the framework presented here treats them as such.

REFERENCES

1. D. A. Close, R. C. Bearse, and H. 0. Men love, "252Cf-Based Hydrogen Analyzer," NuLl. Inst. Meth. 136, 131-135 (1976).

2. M. M. Meier ana E. L. Adams, "HTGR 'Green' Rods Intercomparison: I. Hydrogen Assay," Los Alamo National Laboratory report (to be published).

14 
APPENDIX A

PROGRAM LSQMKI ANU TYPICAL OUTPUT

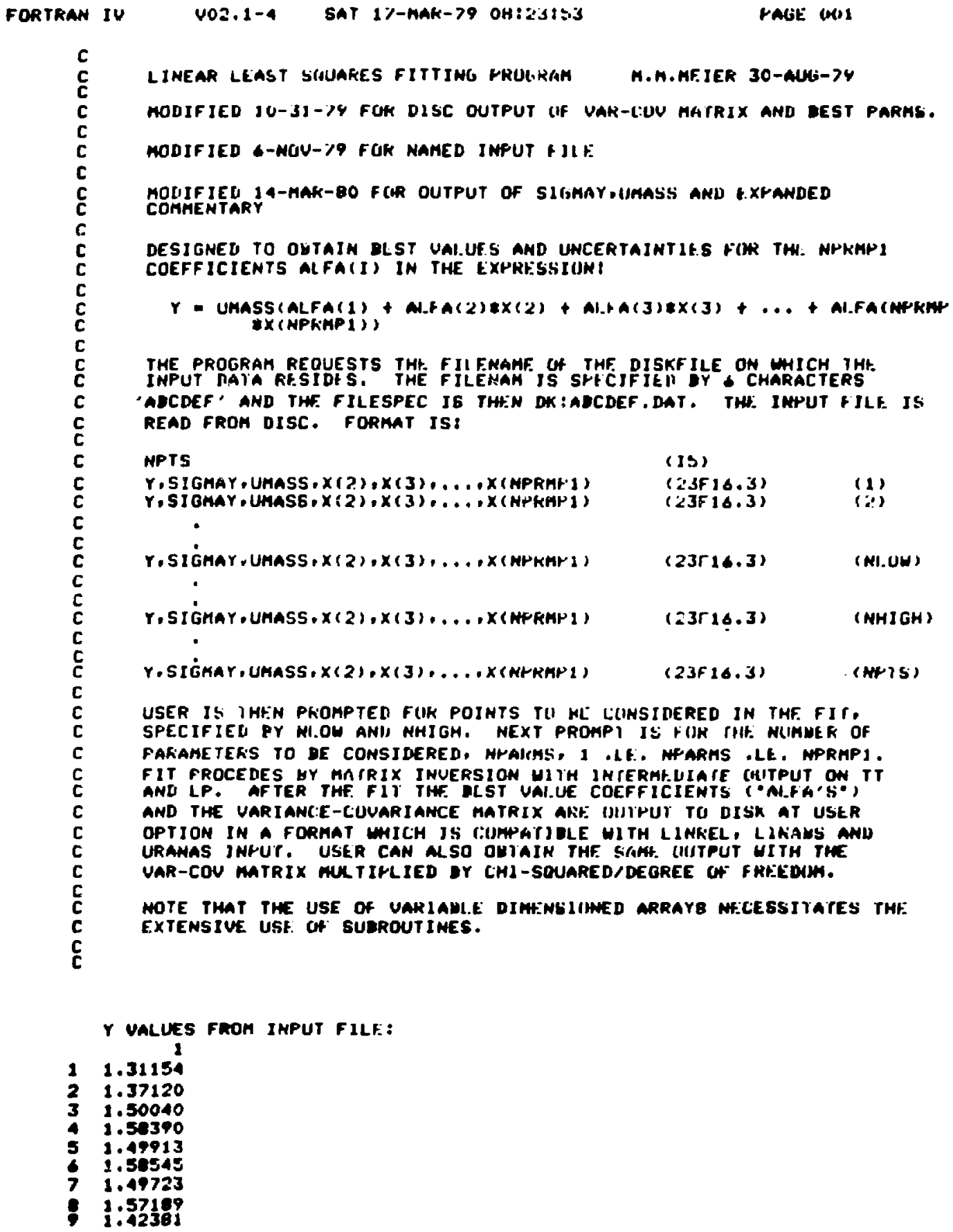


SIOMAY UALUES FROM INFUT FILE:

1

$10.105000 E-02$

$20.114000 E-02$

$30.120000 E-02$

$0.126000 E-02$

$30.119000 E-02$

$60.125000 \mathrm{E} \cdot 02$

$70.1210005 \cdot 02$

$80.125000 E-02$

$0.112000 \mathrm{E}-02$

\section{$X$ UALUES FROM IHFUT FILE:}

$\begin{array}{rrrr}1 & 1 & 2 & 3 \\ 2 & 1.00000 & 0.439600 & 0.000000 \\ 3 & 1.00000 & 0.500400 & 0.000000 \\ 4 & 1.00000 & 0.576900 & 1.00000 \\ 5 & 1.00000 & 0.642000 & 1.00000 \\ 6 & 1.00000 & 0.571300 & 1.00000 \\ 7 & 1.00000 & 0.646700 & 1.00000 \\ 6 & 1.00000 & 0.569600 & 1.00000 \\ 9 & 1.00000 & 0.630600 & 1.00000 \\ & & 0.498300 & 1.00000\end{array}$

MATRIX $=1.000 E+00$

\section{3}

$10.626593 E+070.344578[+070.448944[+07$

$20.344578[+070.195600 E+070.26+201 E+07$

$30.448944 E+070.266201 E+070.448944[+07$

INUERTEI MATRIX $* 1,000 E+00$

$$
1 \text { - } 3
$$

$10.202196 E-04-0.419742 E-040.466892 E-05$

$2-0.1197+1 E-040.897830 E-01-0.122628 E-04$

$30.4668 P 2 E-05-0.112628 E-040.223208 E-05$

PROLUCT OF MATRIX ANI INUEFSE

MAXIMUH DIAGONAL EI.EMENT $=1,00000$ MINIMUA IIAGONAL ELEMENT - $\$ .00000$

MAXIMUM OFF DIAGONAL ELEMFNT:0.121022E-14

\section{Parameter values}

$\begin{array}{lll}10.018304^{1} & A(1) \\ 21.11361 & A(2) \\ 30.466703 E-01 & A(3)\end{array}$

SUH OF RF.SIDUALS FOR EACH MFASURFAH.NI

1

122.3736

214.5860

333.0991

$4 \quad 10.0225$

(5) 2.96469

- 0.595299E-01

72.08675

- 13.9821

- 7.63785

SUM OF ALL RESIDUALS $=\$ 7.6122$

CHI $\$ 2 /$ DEG OF FREEDOH $x(1), \times(2), x(3)$

Variance-covariance matrix

Matrix inversion test 


\section{APPENDIX B}

H MONITOR RESPONSE--A PROGRAM FOR THE HP-67

After entering the -rogram from magnetic card, begin loading the necessary constants into the Hr-57 registers.

Enter

$$
\begin{aligned}
(\Delta O T / D T)^{2} & =\left[\Delta P_{0}(T) / P_{0}(T)\right]^{2}+\left[\Delta P_{0}(1) / P_{0}(1)\right]^{2} \\
& =2\left[B 1(T)+B 2(T)+N_{\text {Lost }}\right] /\left[P_{0}(T)\right]^{2}
\end{aligned}
$$

into register 3. Enter $A 1$ and $A 2$ into registers 4 and $5 ; A 3,2{ }^{\star} A 4$ and $A 5$ into registers 6,7 , and $8 ; P_{0}(1)$, the net pulser are $z$ of the reference run, into register $E$. Enter $N(T)$, "ENTER". Then begin the program by pressing Label "A"; $N(T)$ remains in the display. Enter $T$; "R/S"; $T$ remains in the display. Enter $P(T)$; "R/S"; $P(T)$ remains in the display. Enter $B l(T)$; "R/S"; the difference $P(T)$ - $B 1(T)$ is displayed. Enter $B 2(T)$; "R/S"; $B 2(T)$ remains in display. "R/S"; program runs, then $R(T)$ is displayed. "R/S"; then $R(T)$ is displayed. "R/S"; then $R(T) / R(T)$ is displayed. At this time $R(T), R(T)$, and $R(T) / R(T)$ are stored in registers $A, B$, and $C$ and can be recalled for recording. Calculations for $R(T+1)$ are done by repeating the instructions, beginning with Enter $N(T+1)$, "ENTER". 
APPENDIX C

PROGRAM LINREL AND TYPICAL OUTPUT

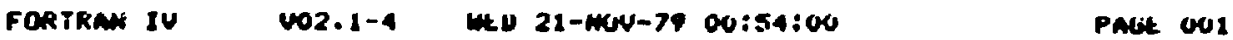

C

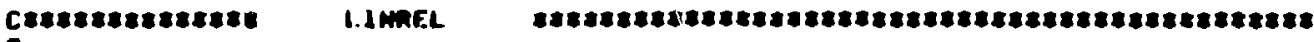

C

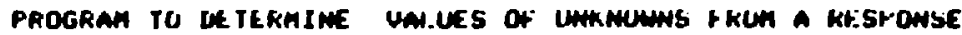

WICM HAS THE FOKM:

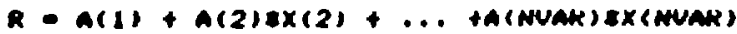

THE Respowse of a stawDaRd hnS Im: SAMf FUKMI

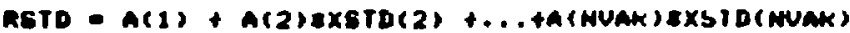

THE A(I)'S AHF. IMPUT FRON A ILE ON LK A.UWG WJ IM IH:IR

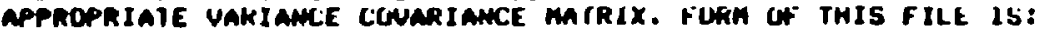

\section{Wuar}

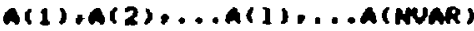

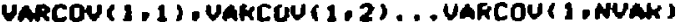

Uancov $(3,1)$, VAKC $(2)(2,2), \ldots$ VAKC CON 2 , AWAK $)$

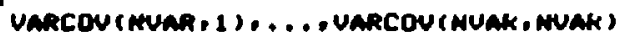

'KUAK - A MMAER O THEFN 2 AND NUAR IS INHIT ID SHIECT THE UMANUWN

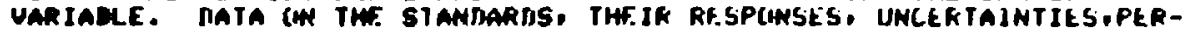
TUREING COASTITUENTS (XSTD(I)'SI AND IINCEKIAINTIES AKH. RHAD PRIH AWUTHER DK FILE UITH THF FOLLOAING FUKMAT (SUBRCUTIME RDRSTDI: mro

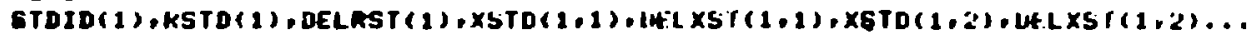
XSTD(I, MUAR) , IH LXST ( I MUAR)

-

-

-

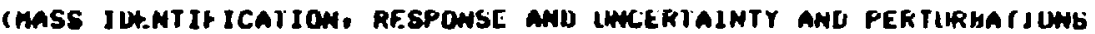
AND THEIK UNCERTAINTIES)

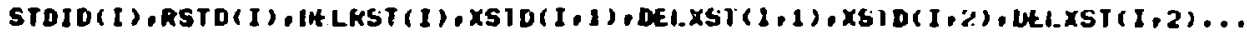
XST D ( I, MUAR ) , WELXGT (I , MUAK)

-

-

STDIDINSTO),RETDCNSTD) ETC.

DATA DW THE SAMPLES. THEIR KF. HUASES. UMCERTAINTIES.PEK-

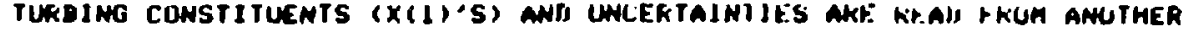

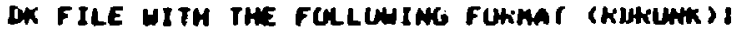
MTS

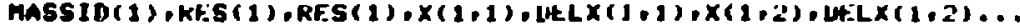

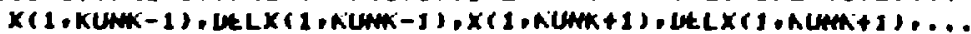

X(1.NYAR-1), IEL X $(1$, HUAR-1)

-

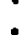

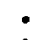

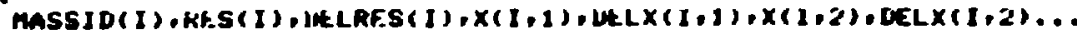

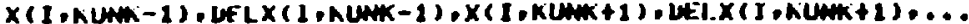
$x(I$. WUaR -1 ) , UFLLX (I . MUAR-1)

-

-

MASSIDUNTSP OKF.S(MPTE) EVC.

"MLON' ANO 'MHJCH' AKF IT IMPUT AND SAMPLES UJTH INDJCES

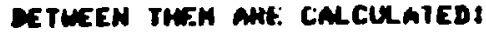




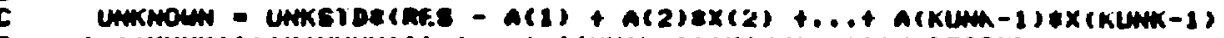

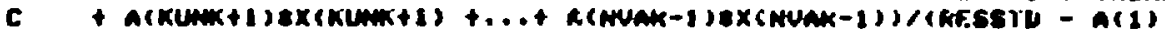

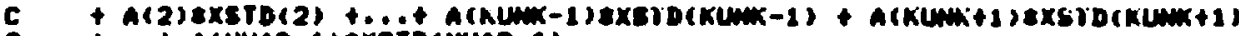

c t...t atmun-1)

C

Castap

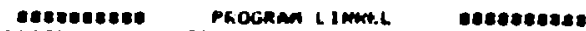

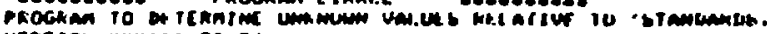
urnstow an: $11-20-70$

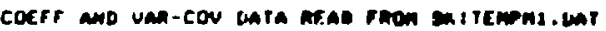

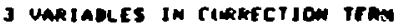

COARECTION COKFFICIENTS

$$
10.0103 E+00
$$

$30.047-01$

LSQMKI

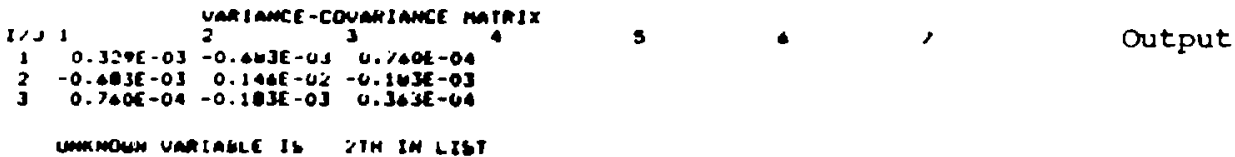

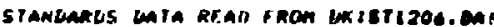

2 standakls in this File.

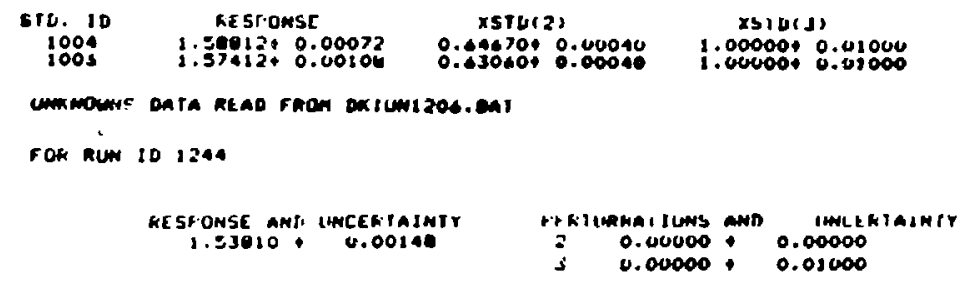

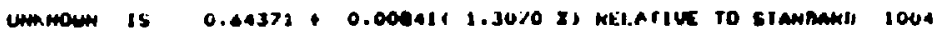

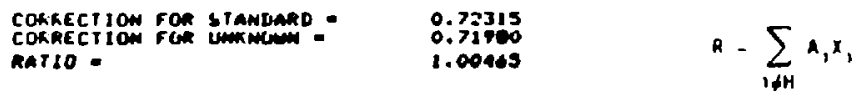

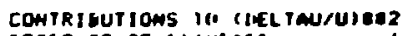



RESPONSE of ummolm -

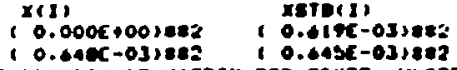

$o\left(R^{S}\right) / O(S)$

$a\left(R^{U}\right) H^{S} /\left[O(S) H^{L}\right]$

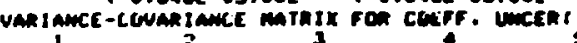



$\begin{array}{llll}1 & 0.32 C E-07 & 0.000 \mathrm{0}+00 & -0.1411-05 \\ 2 & 0.0006+00 & 0.000 \mathrm{0}+00 & 0.0005+00\end{array}$

- 0 . In

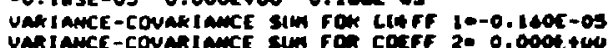

van Iance-covariamce sun for coter 200.0005 two

$\operatorname{varcov}\left(x, A_{j}\right)\left[x_{1}^{U} / 0(0)-x_{i}^{S} / D(S)\right]$

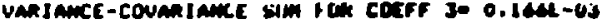

III or nAtRIX - o.1445-eJ

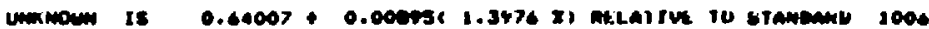

ConkECTION Fon stamene.

conRection for ummine

AAT 20
0.7015

0.710

- $\cos 20$ 


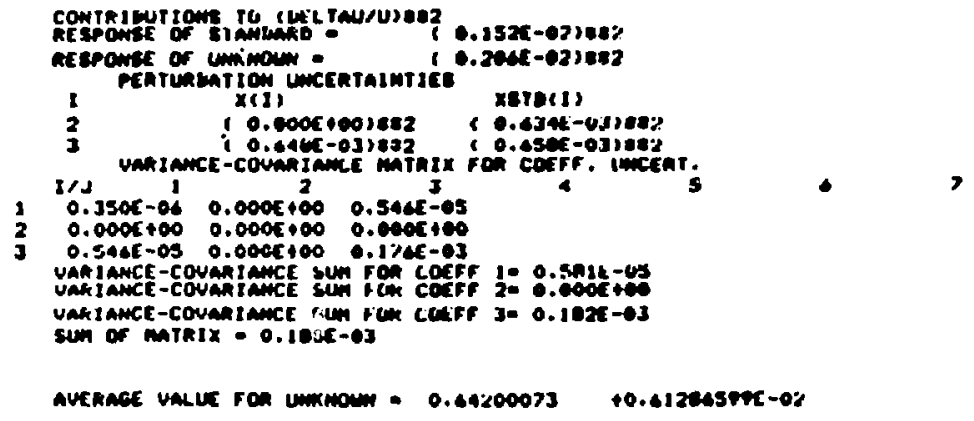

